\title{
Effect of partial sugar replacement with ultrasonically treated citrus pectin on aeration and rheological properties of batter
}

\begin{abstract}
Ultrasound treatment was applied on citrus pectin solution prior mixing into batter as partial replacement of sugar content. The pectin solutions were treated with ultrasound at $20 \%, 40 \%$, and $60 \%$ amplitude for 5, 15, and $25 \mathrm{~min}$. It was then replaced with $20 \%$ and $30 \%$ of sugar content in batter formulation. Aeration properties were tested by measuring batter density and rheological properties of batter, also volume and hardness of cake. The results shown that $20 \%$ of sugar replacement with non-treated pectin in batter system gave lower batter density at lower viscosity, and higher consistency index with less viscoelasticity produced lower cake volume and cake hardness compared with $30 \%$ of sugar replacement. Ultrasound treatment on pectin solution at shorter duration was able to further reduce the batter density which increases the cake aeration by producing higher cake volume with lower hardness compared with non-treated pectin in lower level of sugar replacement.
\end{abstract}

Keyword: Partial sugar replacement; Ultrasonically treated citrus pectin; Aeration; Rheological properties; Batter 